\title{
PEGylated liposomal photosensitizers as theranostic agents for dual-modal photoacoustic and fluorescence imaging-guided photodynamic therapy
}

\author{
Hao $\mathrm{Xu}^{*, \dagger}$, Yubin $\mathrm{Liu}^{\dagger}$, Junle $\mathrm{Qu}^{*,+}$ and Zhen $\mathrm{Yuan}^{\dagger, \S}$ \\ ${ }^{*}$ College of Optoelectronic Engineering \\ Shenzhen University, Key Laboratory of Optoelectronic \\ Devices and Systems of Ministry of Education \\ and Guangdong Province \\ Shenzhen, Guangdong, P. R. China \\ ${ }^{\dagger}$ Faculty of Health Sciences, University of Macau \\ Taipa, Macau SAR 999078, P. R. China \\ †lqu@szu.edu.cn \\ \$zhenyuan@umac.mo
}

Received 3 December 2018

Accepted 20 March 2019

Published 11 April 2019

\begin{abstract}
The photosensitizer (PS) as photodynamic therapy (PDT) agent, can also serve as the contrast agent for dual-modal fluorescence imaging (FLI) and photoacoustic imaging (PAI) for precise cancer theranostics. In this study, the PAI capability of commercial PS, benzoporphyrin derivative monoacid ring- $\mathrm{A}$ (BPD) were examined and compared with that from the other PSs and dyes such as TPPS 4 , Cy5 dye and ICG. We discovered that BPD exhibited its advantage as contrast agent for PAI. Meanwhile, BPD can also serve as the contrast agent for enhanced FLI. In particular, the PEGylated nanoliposome (PNL) encapsulated BPD (LBPD) was produced for contrast enhanced dual-modal FLI and PAI and imaging-guided high-efficiency PDT. Enhanced FLI and PAI results demonstrated the significant accumulation of LBPD both within and among individual tumor during $24 \mathrm{~h}$ monitoring for in vivo experiment tests. In-vitro and in-vivo PDT tests were also performed, which showed that LBPD have higher PDT efficiency and can easily break the blood vessel of tumor tissues as compared to that from BPD. It was discovered that LBPD has great potentials as a diagnosis and treatment agent for dual-modal FLI and PAI-guided PDT of cancer.
\end{abstract}

Keywords: Liposome; photosensitizer; photodynamic therapy; photoacoustic imaging; fluorescence imaging.

This is an Open Access article published by World Scientific Publishing Company. It is distributed under the terms of the Creative Commons Attribution 4.0 (CC-BY) License. Further distribution of this work is permitted, provided the original work is properly cited. 


\section{Introduction}

To date, photodynamic therapy (PDT) has emerged as a promising strategy in the personalized treatment of cancers, which generally utilizes PDT drug (photosensitizer, PS) to induce apoptosis and necrosis of tumor cells through the generated reactive oxygen species (ROS) under light irradiation. ${ }^{1}$ Interestingly, some PSs can also serve as the contrast agent for photoacoustic (PA) and fluorescence imaging. ${ }^{2}$ More importantly, the dual-modal fluorescence and PA imaging-guided PDT enables a more precise spatiotemporal control of the localized therapeutic action, as compared to other therapies such as chemotherapy and radiation therapy. ${ }^{3} \mathrm{PA}$ imaging (PAI) is rapidly growing, largely because PAI can recover both the functional and structural information of biological tissues with high optical contrast, high ultrasound resolution and satisfactory penetration depth. ${ }^{4}$ Meanwhile, among all the molecular imaging modalities, fluorescence imaging (FLI) has been widely used in biomedical fields due to its high sensitivity. ${ }^{5}$ FLI plays an essential role in the study of cellular level events such as gene and protein expressions, cancer early detection and treatment. To take advantages of the complementary information from these two optical imaging modalities, we present an optimized approach that combines FLI and PAT to improve the in vivo imaging sensitivity for cancer theranostics.

However, the PAI capabilities for the representative PSs and dyes have not been systematically examined by using various in vivo and ex-vivo tests. Interestingly, since the energy of pulsed laser in PAI is not sufficient to induce singlet oxygen generation for PS, the toxicity of PS produced during PAI is insufficient to kill the cancer cells. ${ }^{2}$ In addition, when the PS is encapsulated in tumor targeted drug carrier that can accumulate PS in malignant tissues ${ }^{6}$ they basically have no toxicity to normal tissue. $^{7}$ In particular, liposome is one of the most promising drug delivery systems/drug carriers in oncology research and clinical trials. ${ }^{8}$ With the development of liposome preparation technology, PEGylated lipid has been added to the lipid film to form the poly(ethyleneglycol)-modified liposomes (PEGylated liposomes). In particular, polyethylene glycol (PEG) coated nanoliposomes (PEGylated NL, PNL) have shown enhanced passive tumor uptake by enhanced permeability and retention (EPR) effect, ${ }^{9}$ altered biodistribution, prolonged circulation half-life, and eliminated subsequent uptake by the reticuloendothelial. However, it is unclear yet whether the PEGylated liposomal PSs that have the best PA imaging abilities can also serve as the contrast agent for FL imaging.

In this study, the PAI capabilities for various PSs including benzoporphyrin derivative monoacid ring-A (BPD), TPPS, ICG, and Cy5 dye were carefully inspected. In particular, we discovered that among the four PSs, BPD showed that it was the best contrast agent for PAI in the near-infrared window. More importantly, BPD can also serve as the contrast agent for enhanced FLI. And then $\mathrm{BPD}$ was further modified to be entrapped in the lipid bilayer of PNL to produce the liposomal BPD (LBPD), which was carefully characterized regarding its size, zeta potential, and PDT properties. In addition, in vivo FLI and PAI were performed to inspect the dual-modal imaging abilities. Further, the in vitro and in vivo PDT were administrated and compared between BPD and LBPD to access the efficiency of PDT. The results demonstrated that LBPD, as a potential cancer theranostic nanoplatform, can not only work as a PS with good PDT efficiency but also as the contrast agent for enhanced dual model FLI and PAI.

\section{Materials and Methods}

\subsection{Measurements of absorbance spectrum for various PSs}

The absorbance spectrum of different PSs were measured using a spectrophotometer (LAMBDA 750 UV/VIS/NIR, PerkinElmer). The molar extinction coefficient (MEC) of different PSs at their absorbance peaks were determined according to the absorption and concentration standard curves. The phantoms for PA spectrum measurements were prepared with mixed solution of dyes and Agar gel $(2 \%)$ in the ratio of $1: 1 .{ }^{10}$ The PA signals of the phantoms were generated by using a home-made PAI system. ${ }^{11}$ For our PAI system, pulsed light from an Nd:YAG laser with OPO (wavelength range from 680 to $1064 \mathrm{~nm}$; pulse duration: 5-10 ns; frequency rate: $20 \mathrm{~Hz}$; Surelite I-10, Continuum) was used to illuminate the phantom. The transducer (1 MHz central frequency; OLYMPUS NDT) and phantom were immersed into a water tank, in which a rotary stage (T-RSW60A, Zaber) was utilized to rotate the transducer relative to the 
center of the tank. For the present study, the PA spectrum was measured by calculating the PA intensity of phantom at different excitation wavelengths ranging from 680 to $960 \mathrm{~nm}$. The PA spectrum was measured by calculating the PA intensity of phantom at different excitation wavelengths ranging from 680 to $960 \mathrm{~nm}$.

\subsection{Synthesis of nanoliposomes}

1,2-dipalmitoyl-sn-glycero-3-phosphocholine (DPPC), cholesterol and 1,2-distearoyl-sn-glycero3-phosphoethanolamine-N-[amino(polyethylene, glycol)-2000] (DSPE-PEG2000) at a molar ratio of 10:5:1 were mixed in chloroform and then $100 \mathrm{mM}$ BPD in acetone were added to the lipid mixture in a glass tube. The organic solvents were evaporated using a rotary evaporation system to make a thin lipid film. The BPD-containing lipid film was hydrated with PBS. The hydrated solution was then subjected to 10 freeze-thaw cycles $\left(4-45^{\circ} \mathrm{C}\right)$. The dispersions were extruded through polycarbonate membranes $(100 \mathrm{~nm}$ pore size, Avanti Polar Lipids Inc, Alabaster, $\mathrm{AL}$ ) at $42^{\circ} \mathrm{C}$ to form unilamellar vesicles. Unencapsulated $\mathrm{BPD}$ was removed by dialysis overnight against $\mathrm{PBS}$ at $4^{\circ} \mathrm{C}$ using a 300 KD Spectra/Por@ Float-A-Lyzer@ G2 system (Spectrum® Laboratories, Rancho Dominguez, CA).

\subsection{Characterization of nanoliposomes}

The concentration of BPD in liposomal PSs were determined according to its MEC. The BPD leaked out of the stored liposomes was monitored by dialyzing free $\mathrm{BPD}$ in the $300 \mathrm{KD}$ dialysis system at different intervals. The residual concentration of BPD inside the liposomes was determined by measuring their absorbance spectrum after the dialysis. The leakage of BPD from the liposomes was quantified by subtracting the residual concentration from the original concentration. The size, polydispersity index (PDI) and zeta potential of the liposomes were measured by dynamic light scattering with a Zetasizer Nano ZS90 (Malvern Instruments, Malvern, UK).

\subsection{Cell culture and in vitro PDT}

HeLa cell lines were incubated in complete Dulbecco's Modified Eagle Medium (DMEM) (containing $10 \%(\mathrm{v} / \mathrm{v})$ fetal bovine serum and $1 \%$ $(\mathrm{v} / \mathrm{v}) 5000$ I.U. per $\mathrm{mL}$ penicillin/streptomycin) at $37^{\circ} \mathrm{C}$ in a $5 \% \mathrm{CO}_{2}$ incubator. Before cell toxicity measurement, HeLa cells were plated in $35 \mathrm{~mm}$ cell culture dishes and incubated overnight at $37^{\circ} \mathrm{C}$ under $5 \% \mathrm{CO}_{2}$. Then attached cells were incubated with free BPD or LBPD according to the experimental concentration in culture media for $90 \mathrm{mins}$ under subdued light conditions, subsequently refeed with $2 \mathrm{~mL}$ of fresh culture media, and further exposed to a laser irradiation system. In this system, light was delivered from a $690 \mathrm{~nm}$ fiber-coupled diode laser (MRL-III-690, Changchun New Industries Optoelectronics Technology Co.), which were connected to the optical fiber. The laser power $50 \mathrm{~mW} / \mathrm{cm}^{2}$ was determined with a laser power energy meter (FieldMate, Coherent). Dark toxicity of BPD and control groups were respectively prepared in the same way without laser irradiation. All dishes were then incubated for $24 \mathrm{~h}$ at $37^{\circ} \mathrm{C}$ under $5 \% \mathrm{CO}_{2}$. Cell survival was assessed on the second day using commercial CCK-8 cell viability test. CCK-8 kits (Dojindo, Japan) containing a watersoluble tetrazolium salt (WST-8) can be reduced to a yellow color formazan dye by dehydrogenase activities. ${ }^{12}$ All cell culture plates were refeeded with $1 \mathrm{~mL}$ completed cell culture medium containing $100 \mu \mathrm{L}$ CCK-8 solution. Finally, three-fold replicates were run and cell survival was estimated by the absorption of every well at $450 \mathrm{~nm}$ using a

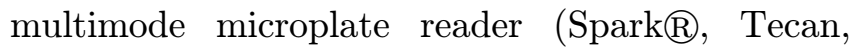
Switzerland). All experiments were repeated at least three times.

\subsection{In vivo tumor implantation}

All animal experiments were performed in compliance with the guidelines on animal research stipulated by the Animal Care and Use Committee at the University of Macau. The female BALB/c nude mouse (The Jackson Laboratory, USA) were cared under dark and aseptic conditions in a small animal facility. The tumor implantation was prepared by subcutaneously injecting a $50 \mu \mathrm{l}$ PBS (contained $10^{7}$ HeLa cells) on the surface of hind leg.

\subsection{In vivo and ex vivo FLI}

Before FLI, $0.2 \mathrm{~mL}$ LIT (contained $0.2 \mathrm{mg} \mathrm{kg}^{-1}$ $\mathrm{BPD})$ was injected through the tail vein. All mice 
were anesthetized by isoflurane during imaging. The biodistribution of LBPD was accessed by in vivo FLI at different time points post-injection. And then the mice were sacrificed after $24 \mathrm{~h}$, in which the major organs and tumors were immediately extracted and imaged to quantify the distribution of LBPD inside them ex vivo. In vivo and ex vivo fluorescence images were acquired using an in vivo imaging system (IVIS Lumina XRMS, Bruker) by setting $450 \mathrm{~nm}$ excitation filter (band-pass $\pm 10 \mathrm{~nm}$ ) and $710 \mathrm{~nm}$ emission filter (band-pass $\pm 17.5 \mathrm{~nm}$ ). The photography, X-ray and fluorescence images were generated at the same time points.

\subsection{In vivo $P A I$}

$0.2 \mathrm{~mL}$ BPD (contained $0.2 \mathrm{mg} \mathrm{kg}^{-1} \mathrm{BPD}$ ) was injected through the tail vein. The tumored nude mice were anesthetized by isoflurane and fixed on a holder. The tumor volumes were immersed into a water tank with warm water. The same home-made PAI system for the phantom tests was applied to animal in vivo tests.

\subsection{In vivo $P D T$}

To estimate the efficacy of PDT, the vascular structure changes of LBPD injected mice ear during PDT were accessed with our home-made opticalresolution photoacoustic microscopy (PAM) system. ${ }^{13}$ For the PAM system, the pulsed laser (wavelength: $532 \mathrm{~nm}$; pulse width: $1 \mathrm{~ns}$; and repetition rate: up to $5 \mathrm{KHz}$ ) was used to illuminate the animals. The light beam first went through an optical subsystem (NDF: neutral density filter; CL1 and CL2: convex lens; PH: pinhole; OF: optical fiber), which was subsequently coupled with a single-mode fiber. Then the laser beam delivered by the fiber changed its transportation direction by a rectangular prism (RP) after passing through an objective lens (OL1). To generate a high-resolution PA image, the second objective lens (OL2) was utilized, by which the laser beam can be focused on the imaging areas. A two-dimensional motor (M1 and M2) stage was rotated to scan the mouse ear. The mouse ear was fixed upon the agar gel phantom, the ultrasonic transducer was placed under the phantom, and a thin layer of ultrasound gel was spread over the interface between the agar phantom and transducer. The PA signals detected by a $50 \mathrm{MHz}$ transducer with an element diameter of
$5.08 \mathrm{~mm}$ and a focal length of $127 \mathrm{~mm}$ (VF 412, Valpey Fisher) were amplified by the amplifier (5073R, OLYMPUS) and collected on the computer to generate optical-resolution PAM images. ${ }^{14}$

\subsection{Statistical analysis}

All experiments were performed in triplicate and the data were expressed as mean plus and minus the standard deviation (SD). Student's $t$-test were used to determine the statistically significant differences between different groups when appropriate.

\section{Results and Discussion}

\subsection{Absorbance and photoacoustic spectrum of different PSs}

To quantify the optical absorbance of these PSs, the wavelength-dependent absorption spectra of each PS with different concentrations were measured by a spectrophotometer. As shown in Fig. 1, porphyrin-based photosensitziers including BPD and $\mathrm{TPPS}_{4}$ exhibited the highest absorbance peak range for wavelegnths between 400 and $500 \mathrm{~nm}$. This wavelength band can be used as the fluorescence excitation wavelenght for FLI, whereas the PSs with wavelegnths higher than $600 \mathrm{~nm}$ can serve as the agents for PDT. By contrast, PAI is generally performed in the near-infrared window with illumination wavelength higher than $600 \mathrm{~nm}$. Interestingly, we discovered that the absorbance peak of BPD,TPPS 4 , Cy5 dye, and ICG are 690, 646, 650, and 794 , respectively.

However, as shown in Figs. 2(a) and 2(b), BPD at $698 \mathrm{~nm}$ exhibited the highest PA intensity and the best PAI capabilties as compared to those from Cy5, ICG, and $\mathrm{TPPS}_{4}$ for the whole near-infrared window. Since BPD has the highest absorbance at $690 \mathrm{~nm}$, it can serve as the contrast agent for enhanced PAI with the irradiation wavelength at $698 \mathrm{~nm}$. In addition, the linear relationship between various $\mathrm{BPD}$ concentrations and $\mathrm{PA}$ intensities were also confirmed and the results were provided in Fig. S2.

\subsection{Synthesis and characterization of $L B P D$}

LBPD were fabricated by entrapping BPD in the lipid bilayer of the sterically stabilized moderately 


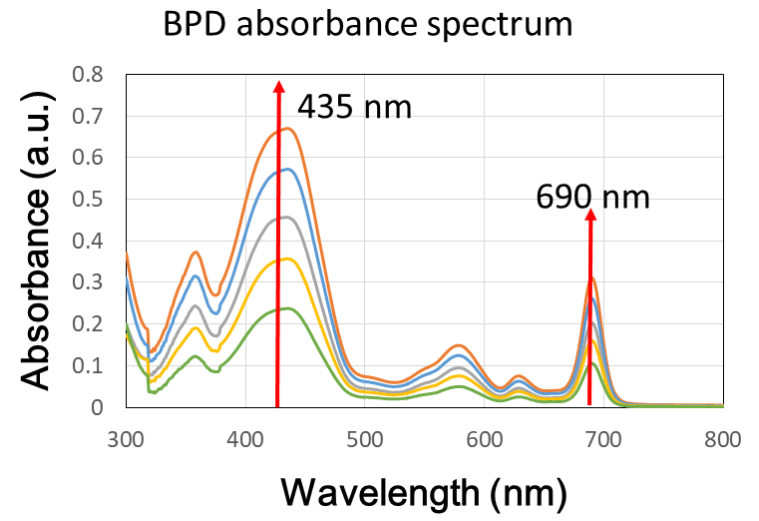

(a)

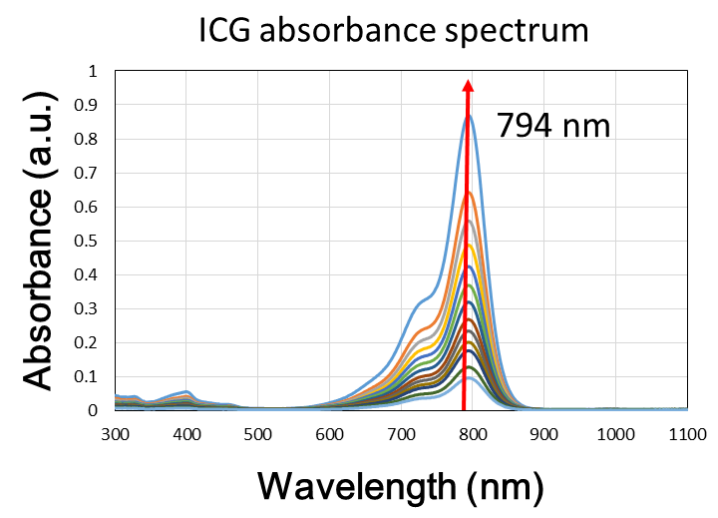

(c)

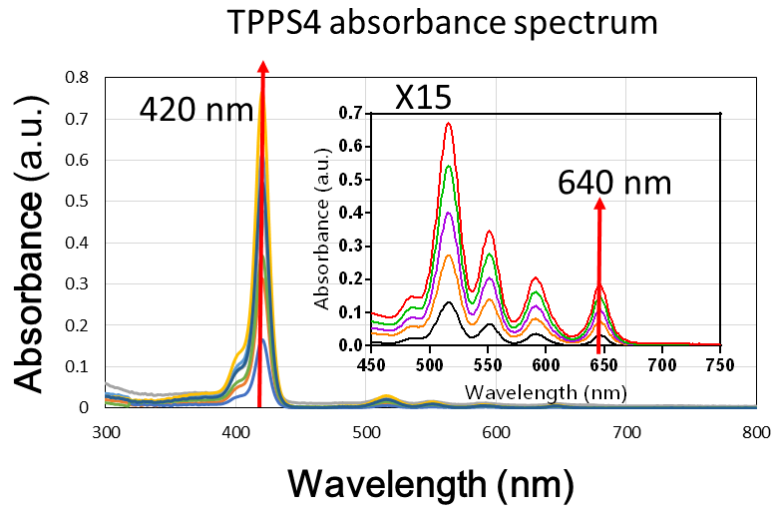

(b)

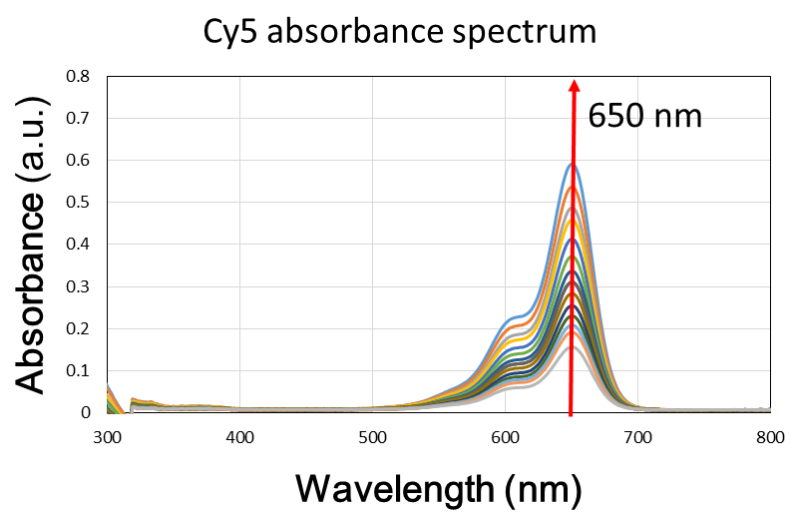

(d)

Fig. 1. Absorption spectra of (a) BPD, (b) $\mathrm{TPPS}_{4}$, (c) ICG and (d) Cy5 at different concentration. The insert absorption spectra in (b) is a 15-fold higher concentration than the original concentration of $\mathrm{TPPS}_{4}$.

cationic PEGylated liposomes. Figure 3(a) showed the structure of LBPD, in which cholesterol was added to the NL formulation to modulate membrane permeability and biological stability. ${ }^{15}$ Meanwhile, DSPE-PEG2000 was doped in the lipid

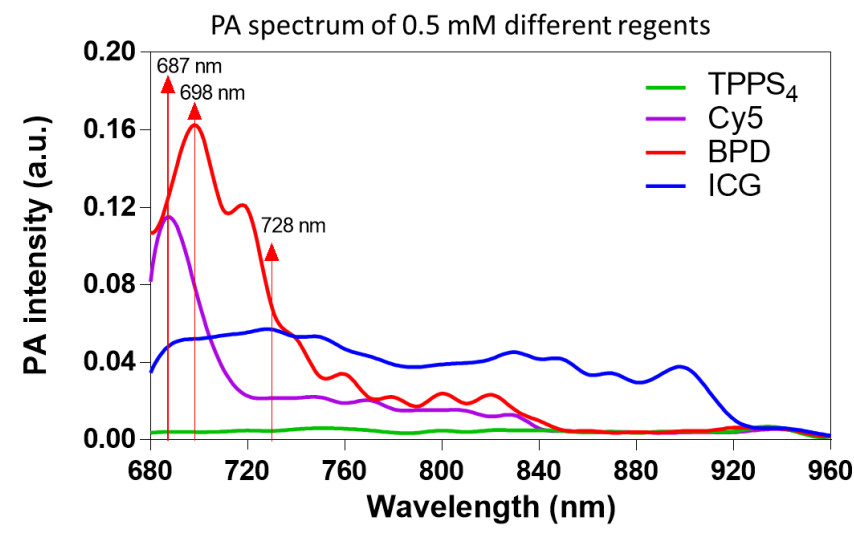

(a) film to form the PEGylated liposomes, which can alter the biodistribution, prolong the circulation half-life, and avoid the recognition and subsequent assimilation by the reticuloendothelial system (RES). ${ }^{16}$ The characterization of LBPD including

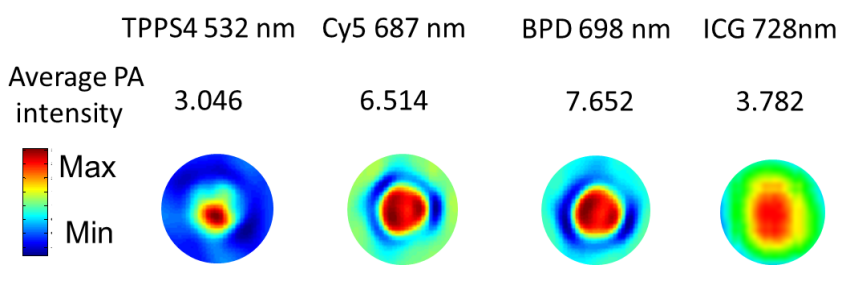

(b)

Fig. 2. (a) Photoacoustic spectrum of $\mathrm{TPPS}_{4}, \mathrm{Cy} 5$, BPD and ICG. (b) Phantom imaging of TPPS 4 , Cy5, BPD and ICG. 


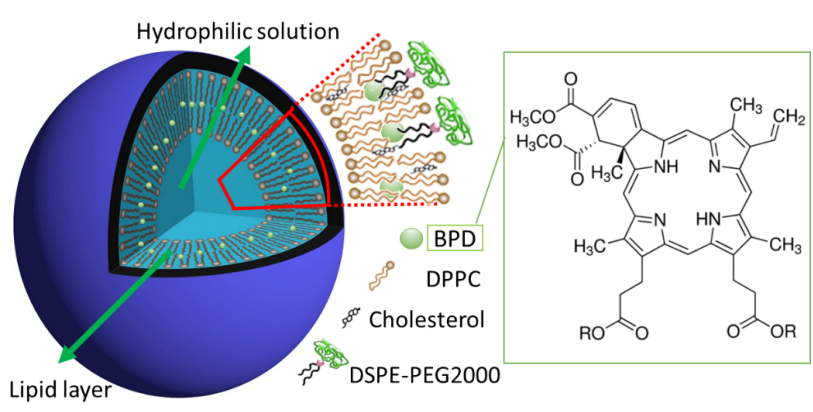

(a)

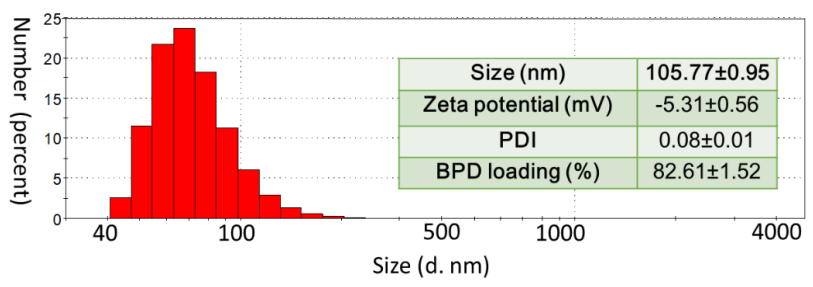

(b)

Fig. 3. (a) The schematic diagram illustrating LBPD. (b) The size distribution and the obtained characteristics (average size, average zeta-potential and percentage of the loaded BPD).

the size distribution, average size, average zetapotential, and percentage of the loaded BPD) was performed and the results were provided in Fig. 3(b). The average diameter of LBPD was found to be $105.8 \mathrm{~nm}$. The size distribution of LBPD exhibited that the diameter of most of the fabricated LBPD ranges between 50 and $100 \mathrm{~nm}$, which is appropriate for enhanced permeation through the leaky tumor vasculature. ${ }^{17}$

The concentration of BPD in the LBPD was estimated using the known absorptivity (molar extinction coefficient) of BPD in DMSO solution (Fig. S1). As a result, liposomal formulations were dissolved in DMSO to break nanoliposome (NL) and release $\mathrm{BPD}$, and then the absorption spectra were acquired and compared to that from free BPD in DMSO.

The BPD loading efficiency in LBPD was determined as a percentage of the PS encapsulated in the NL of the whole PS amount used for the NL preparation. As listed in Fig. 3(b) inserted table, the BPD loading efficiency in LBPD was around $82.6 \pm 1.5 \%$.

\subsection{In vitro PDT}

The cell viabilities were assessed with Cell Counting Kit-8 (CCK-8) $24 \mathrm{~h}$ after treatment with free BPD or LBPD. As shown in Fig. 4, no significant dark toxicity was revealed for HeLa cells incubated with free or BPD and no HeLa cell death was identified under the laser irradiation $\left(10 \mathrm{~J} \mathrm{~cm}^{-2}\right)$ at $690 \mathrm{~nm}$ for the control (untreated) groups. By contrast, the cell survival percentage in the free BPD and $\mathrm{LBPD}$ group was $29.4 \%$ and $26.9 \%$, respectively, under laser irradiation at $690 \mathrm{~nm}$, which indicated that the LBPD and BPD have similar PDT efficiency. The free BPD was dissolved in DMSO firstly due to its hydrophobic characters. Although the concentration of DMSO is very low, it is still toxic to the cells, whereas the LBPD was dissolved in PBS, which almost has no toxicity for cells. In addition, LBPD have better in vivo tumor targeting and retention, which is superior for imaging and monitoring the PDT treatment.

\subsection{In vivo and ex vivo FLI of $L B P D$}

To examine the in vivo FLI capability and tumor targeting ability, LBPD was injected intravenously into tumor-bearing BALB/c mice. As displayed in Fig. 5(a), the fluorescence images of mice were generated at different post-injection time points and were then merged with the corresponding high-resolution X-ray images which were taken at the same time point to serve as a background image. As plotted in Fig. 5(a), enhanced FL signals were

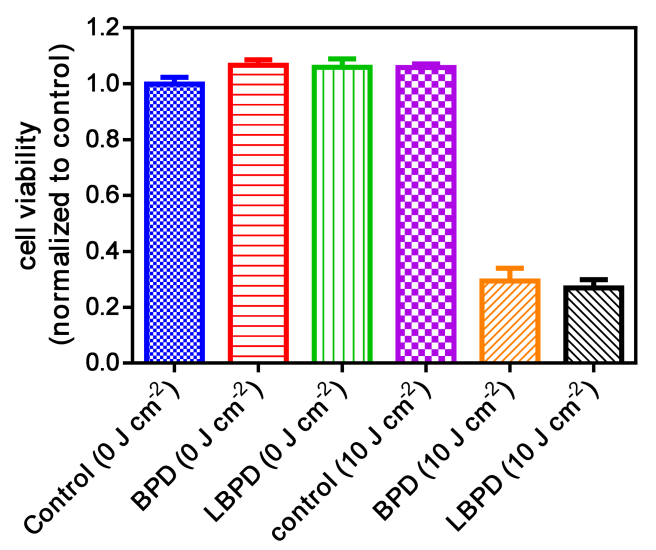

Fig. 4. HeLa cells survival 1 day post-PDT. PDT experiments were performed in triplicate and the data were expressed as mean plus and minus the SD. 


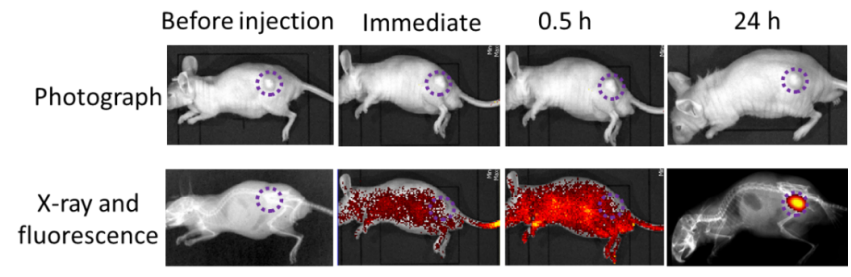

(a)

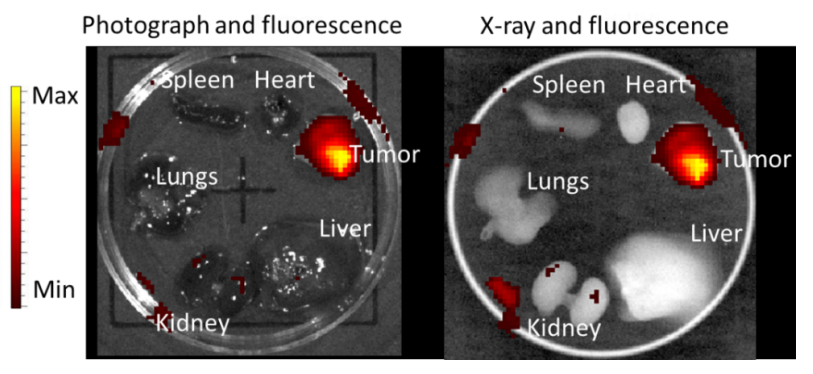

(b)

Fig. 5. (a) X-ray and fluorescence images of the tumored nude mouse at before injection and various time points post injection with LBPD (immediate, $0.5 \mathrm{~h}$, and $24 \mathrm{~h}$ ). Purple circles indicate the tumor tissue. (b) Photograph and fluorescence images of the major organs resected from mouse $24 \mathrm{~h}$ post injection.

identified in the tumor region (circled in purple) at $24 \mathrm{~h}$ post-injection. The FLI results indicated that BPD can accumulate in the tumor within a longer time period ( $24 \mathrm{~h}$ post-injection), which can be attributed to the prolonged circulation time for the PEGylated liposomes ${ }^{8}$ and the related improvement of the passive tumor targeting due to EPR effect. ${ }^{18}$

After $24 \mathrm{~h}$ post-injection of LBPD, mice were sacrificed and the organs were extracted, resected and imaged immediately. The ex vivo fluorescence images were provided in Fig. 5(b). We discovered from Fig. 5(b) that the FLI results of major organs validated the accumulation of LBPD mainly in the tumor, and the low FL signals from other organs indicated a high efficiency of the passive tumor targeting with LBPD.

\subsection{In vivo $P A I$ of $L B P D$}

The tumor-bearing mice were intravenously injected with free BPD and LBPD, respectively. And then PAI was performed at wavelength $690 \mathrm{~nm}$. As shown in Fig. 6, PA signal showed no change at tumor site after the injection of free BPD. However, this is not the case for injection of LBPD, in which the PA signals were significantly enhanced at $6 \mathrm{~h}$ post-injection, and the PA intensity was remarkably increased in the tumor tissue at $24 \mathrm{~h}$ postinjeciton. The PAI results demonstrated that liposome encapsulated BPD (LBPD) has better tumor accumulation and retention than the free BPD due to the PEGylated strategy in the liposome preparation. The imaging results also indicated that LBPD can serve as PA contrast regent for monitoring the regent accumulation in the tumor tissue.

\subsection{In vivo PAI of vascular structure changes during PDT}

To insepct the PDT efficiency of LBPD, the mice ear injected with LBPD was photoacoustically imaged before and after PDT treatment. As shown in Fig. 7, the vascular structure changes including the significant vessel damage was identified with $10 \mathrm{~J} \mathrm{~cm}^{-2}$ laser irradiation at $690 \mathrm{~nm}$. These PAI results demonstrated that PDT with LBPD as PS can damage the vascular networks of tumor under laser irradiation. The occlusion of the vasculature

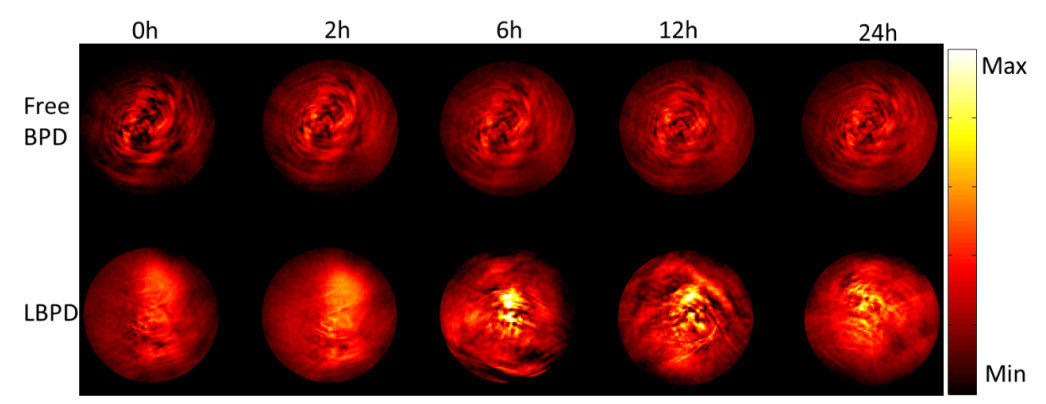

Fig. 6. PA images of the tumor sites at different time point with free BPD and LBPD under $698 \mathrm{~nm}$ pulse laser with the incident optical fluence of $10 \mathrm{~mJ} \mathrm{~cm}^{-2}$. 

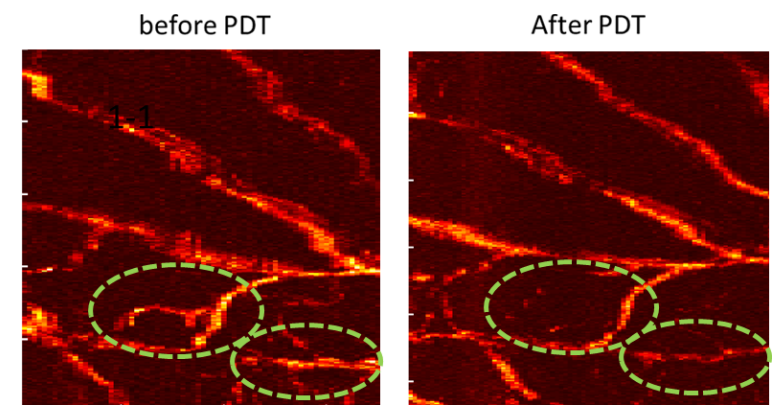

Fig. 7. Photoacoustic images of vascular structures before and after PDT. The green circles indicate the significant changes of the vascular structures.

will decrease the nutrient supply of the tissue, which is essential for PDT. ${ }^{19}$

\section{Conclusions}

Advances in molecular imaging and NL have enabled us to design novel theranostic nanoplatforms. The PS encapsulating nanoliposomes was designed for the present study for dual-model FLI and PAIguided PDT. The LBPD as a robust PS exhibited its unbeatable advantages in serving the contrast agent for enhanced PAI and FLI. More importantly, the developed nanoplatform is a clinically approved PS in liposomal encapsulation, which is the most popular clinically accepted nanosized drug delivery vehicles. ${ }^{8}$ This multifunctional theranostic regent has exhibited its potential for clinical translatability. Further study should involve the evaluation of LBPD-based preclinical therapy to realize the dual-modal imaging guided precision PDT.

\section{Conflict of Interest}

The authors have declared that no competing interest exists.

\section{Acknowledgments}

This work was supported by the National Basic Research Program of China (2015CB352005), the National Natural Science Foundation of China (61705139, 61875135, 61525503, 61620106016, 61835009, 81727804), Guangdong Natural Science Foundation Innovation Team (2014A030312008, 2017A030310136), Shenzhen Basic Research Project (JCYJ20150930104948169, JCYJ20160328144
746940， GJHZ20160226202139185， JCYJ2017041 2105003520，JCYJ20170817094735945，JCYJ201 70818090620324), China and Postdoctoral Science Foundation (2017M612724), China. This study was also supported by MYRG2016-00110-FHS and MYRG grants from the University of Macau in Macau, and FDCT 0011/2018/A1 and FDCT 025/ 2015/A1 grants from Macao government.

\section{References}

1. J. P. Celli et al., "Imaging and photodynamic therapy: Mechanisms, monitoring, and optimization," Chem. Rev. 110, 2795-2838 (2010).

2. A. B. E. Attia et al., "Phthalocyanine photosensitizers as contrast agents for in vivo photoacoustic tumor imaging," Biomed. Opt. Exp. 6, 591-598 (2015).

3. T. Hasan et al., "Photodynamic therapy of Cancer," Holland-Frei Cancer Medicine D. W. Kufe et al. Eds., pp. 605-622 B.C. Decker, (2003).

4. J. Zhang et al., "A PIID-DTBT based semiconducting polymer dots with broad and strong optical absorption in the visible-light region: Highly effective contrast agents for multiscale and multispectral photoacoustic imaging," Nano Res. 10, 64-76 (2017).

5. J. Cheon, J. H. Lee, "Synergistically integrated nanoparticles as multimodal probes for nanobiotechnology," Acc. Chem. Res. 41, 1630-1640 (2008).

6. D. Kumar, L. Shan, Y. Zhang, "Nanoparticles in photodynamic therapy: An emerging paradigm," Adv. Drug Deliv. Rev. 60, 1627-1637 (2008).

7. S. Lucky et al., "Nanoparticles in photodynamic therapy," Chem. Rev. 115, 1990-2042 (2015).

8. G. Obaid et al., "Photonanomedicine: A convergence of photodynamic therapy and nanotechnology," Nanoscale 8, 12471-12503 (2016).

9. H. Kobayashi et al., "Improving conventional enhanced permeability and retention (EPR) effects; What is the appropriate target?" Theranostics 4, 81-89 (2014).

10. Y. Liu, H. Jiang, Z. Yuan, "Two schemes for quantitative photoacoustic tomography based on Monte Carlo simulation," Med. Phys. 43, 3987-3997 (2016).

11. D. Gao et al., "Protein-modified $\mathrm{CuS}$ nanotriangles: A potential multimodal nanoplatform for in vivo tumor photoacoustic/magnetic resonance dual-modal imaging," Adv. Health. Mater. 6, (2017).

12. H. Tominaga et al., "A water-soluble tetrazolium salt useful for colorimetric cell viability assay," Anal. Commun. 36, 47-50 (1999). 
13. J. Zhang et al., "Development of a multi-band photoacoustic tomography imaging system based on a capacitive micromachined ultrasonic transducer array," Appl. Opt. 56, 4012-4018 (2017).

14. D. Y. Gao et al., "Engineering a protein-based nanoplatform as an antibacterial agent for light activated dual-modal photothermal and photodynamic therapy of infection in both the NIR i and II windows," J. Mater. Chem. B 6, 732-739 (2018).

15. S. Vemuri et al., "Preparation and characterization of liposomes as therapeutic delivery systems: A review," Pharm. Acta Helv. 70, 95-111 (1995).

16. M. F. Oliveira et al., "Strategies to target tumors using nanodelivery systems based on biodegradable polymers, aspects of intellectual property, and market," J. Chem. Biol. 6, 7-23 (2013).

17. A. S. L. Derycke et al., "Liposomes for photodynamic therapy," Adv. Drug Deliv. Rev. 56, 17-30 (2004).

18. H. Maeda, "Macromolecular therapeutics in cancer treatment: The EPR effect and beyond," J. Control. Release 164, 138-144 (2012).

19. A. M. Richter et al., "Photosensitising potency of structural analogues of benzoporphyrin derivative (BPD) in a mouse tumour model," Br. J. Cancer 63, 87-93 (1991). 